\title{
Systematic Variations of CO Gas Abundance with Radius in Gas-rich Protoplanetary Disks
}

\author{
Ke Zhang ${ }^{1,5}$ (D), Edwin A. Bergin ${ }^{1}$ (D), Kamber Schwarz ${ }^{2,6}$ (D), Sebastiaan Krijt ${ }^{3,5}$ (D), and Fred Ciesla ${ }^{4}$ (D) \\ ${ }^{1}$ Department of Astronomy, University of Michigan, 323 West Hall, 1085 S. University Avenue, Ann Arbor, MI 48109, USA; kezhang@umich.edu \\ ${ }^{2}$ Lunar and Planetary Laboratory, University of Arizona, 1629 E. University Boulevard, Tucson, AZ 85721, USA \\ ${ }^{3}$ Department of Astronomy/Steward Observatory, The University of Arizona, 933 North Cherry Avenue, Tucson, AZ 85721, USA \\ ${ }^{4}$ Department of the Geophysical Sciences, The University of Chicago, 5734 South Ellis Avenue, Chicago, IL 60637, USA \\ Received 2019 April 29; revised 2019 July 22; accepted 2019 August 4; published 2019 September 24
}

\begin{abstract}
$\mathrm{CO}$ is the most widely used gas tracer of protoplanetary disks. Its abundance is usually assumed to be an interstellar ratio throughout the warm molecular layer of the disk. But recent observations of low CO gas abundance in many protoplanetary disks challenge our understanding of physical and chemical evolutions in disks. Here we investigate the $\mathrm{CO}$ abundance structures in four well-studied disks and compare their structures with predictions of chemical processing of $\mathrm{CO}$ and transport of $\mathrm{CO}$ ice-coated dust grains in disks. We use spatially resolved CO isotopologue line observations and detailed thermo-chemical models to derive $\mathrm{CO}$ abundance structures. We find that the $\mathrm{CO}$ abundance varies with radius by an order of magnitude in these disks. We show that although chemical processes can efficiently reduce the total column of $\mathrm{CO}$ gas within $1 \mathrm{Myr}$ under an ISM level of cosmic-ray ionization rate, the depletion mostly occurs at the deep region of a disk. Without sufficient vertical mixing, the surface layer is not depleted enough to reproduce the weak $\mathrm{CO}$ emissions observed. The radial profiles of CO depletion in three disks are qualitatively consistent with predictions of pebble formation, settling, and drifting in disks. But the dust evolution alone cannot fully explain the high depletion observed in some disks. These results suggest that dust evolution may play a significant role in transporting volatile materials and a coupled chemical-dynamical study is necessary to understand what raw materials are available for planet formation at different distances from the central star.
\end{abstract}

Key words: astrochemistry - circumstellar matter - molecular data - protoplanetary disks

\section{Introduction}

Planets are formed of raw materials in protoplanetary disks around young stars (Hayashi 1981; Williams \& Cieza 2011). The mass distribution and composition of solids and gases in natal disks profoundly affect outcomes of planetary systems, from planetary masses to compositions of planetary cores and atmospheres (Pollack et al. 1996; Öberg et al. 2011; Benz et al. 2014). Recently, high spatial resolution observations of dust continuum and gas lines in disks started providing direct constraints to ongoing planet formation processes (ALMA Partnership et al. 2015; Andrews et al. 2016, 2018). To accurately constrain initial conditions and intermediate processes of planet formation, we need a robust understanding of the physical and chemical processes relevant to observational tracers (Bergin \& Williams 2018).

Among all gaseous tracers of protoplanetary disks, $\mathrm{CO}$ is the most widely detected and studied molecule. It has the advantages of being highly abundant, relatively chemically stable, and readily detectable (Molyarova et al. 2017). The typical view of $\mathrm{CO}$ gas distribution in protoplanetary disks is based on two concepts: the snowline and the warm molecular layer (Aikawa et al. 2002). Along the radial direction, CO exists as the gas inside its snowline and freezes out as ice beyond the snowline. In the vertical direction, the $\mathrm{CO}$ gas presents in a middle layer, the so-called warm molecular layer, of which the lower boundary is where $\mathrm{CO}$ freezes out, and the upper boundary is where $\mathrm{CO}$ is photodissociated by stellar or external radiation. Inside the warm molecular layer, the $\mathrm{CO}$ gas

\footnotetext{
5 Hubble Fellow.

6 Sagan Fellow.
}

abundance is assumed to be a constant across all radii, typically at an interstellar ratio of $\sim 10^{-4}$. This simple parameterization has been widely used in constraining basic properties of protoplanetary disks, such as the total disk mass (Williams \& Best 2014; Ansdell et al. 2016), gas disk radius (Andrews et al. 2012; Ansdell et al. 2018), gas mass distribution (Williams \& McPartland 2016; Zhang et al. 2017; Miotello et al. 2018), gas temperature (Salyk et al. 2009; Flaherty et al. 2015; Schwarz et al. 2016), and kinematic properties due to turbulence and planet-disk interactions (Flaherty et al. 2015; Pinte et al. 2018b; Teague et al. 2018a).

However, this simple picture of $\mathrm{CO}$ in disks has been challenged by growing numbers of observations. Systematic (sub)millimeter surveys of Class II disks in nearby star formation regions showed that the $\mathrm{CO}$ gas-to-dust mass ratios in the majority of Class II disks are one order of magnitude too low compared to the interstellar ratio, even after correcting the effects of $\mathrm{CO}$ freeze-out and isotope-selective photodissociation (Ansdell et al. 2016; Barenfeld et al. 2016; Miotello et al. 2016; Long et al. 2017).

It is still under debate whether the weak CO lines are due to gas dissipation or a subinterstellar $\mathrm{CO}$ abundance, but the latter case has received circumstantial support. All three disks with independent gas mass measurements from HD line fluxes show a significant depletion in $\mathrm{CO}$ abundance (by a factor of 5-100) while their hydrogen gas is still abundant (Bergin et al. 2013; Favre et al. 2013; McClure et al. 2016; Schwarz et al. 2016; Zhang et al. 2017). Also, the measured mass accretion rates and expected ages imply an ISM level gas-to-dust ratio of 100 for disks in the Lupus star formation region (Manara et al. 2016). Furthermore, no correlation was found between the $\mathrm{CO}$ derived 
Table 1

Observation Log

\begin{tabular}{|c|c|c|c|c|c|c|c|}
\hline Source & Project ID & PI & $\begin{array}{c}t_{\text {int }} \\
\text { (minute) }\end{array}$ & $\begin{array}{l}\text { Baseline } \\
\text { (m) }\end{array}$ & Antenna & UT Time & $\begin{array}{c}\text { Channel Width } \\
(\mathrm{kHz})\end{array}$ \\
\hline DM Tau & 2015.1.00308.S & E. A. Bergin & 41 & $15-1091$ & 40 & 2016 Jul & 121 \\
\hline TW Hya & 2016.1.01495.S & H. Nomura & 52 & $15-704$ & 45 & 2016 Dec & 141 \\
\hline HD 163296 & 2013.1.00601.S & A. Isella & 151 & $35-1574$ & 44 & 2015 Aug & 31 \\
\hline \multirow[t]{2}{*}{ IM Lup } & 2013.1.00226.S & K. Oberg & 21 & $20-650$ & 31 & 2015 Jul & 61 \\
\hline & 2013.1.00798.S & C. Pinte & 37 & $21-784$ & 36 & 2015 Jun & 35 \\
\hline
\end{tabular}

Table 2

Line Information

\begin{tabular}{|c|c|c|c|c|c|c|}
\hline Source & Line & $\begin{array}{c}\Delta v \\
\left(\mathrm{~km} \mathrm{~s}^{-1}\right)\end{array}$ & $\begin{array}{c}\mathrm{rms} \\
(\mathrm{mJy} / \text { beam })\end{array}$ & $\begin{array}{c}F_{\text {int }} \\
\left(\mathrm{Jy} \mathrm{km} \mathrm{s}^{-1}\right)\end{array}$ & $\begin{array}{c}\text { Beam } \\
(\operatorname{arcsec}, \text { PA) }\end{array}$ & $\begin{array}{c}\text { Beam } \\
(\mathrm{au})\end{array}$ \\
\hline DM Tau & $\mathrm{C}^{18} \mathrm{O}(3-2)$ & 0.22 & 5 & $3.0 \pm 0.1$ & $0.36 \times 0.29(-47)$ & $50 \times 41$ \\
\hline \multirow[t]{2}{*}{ TW Hya } & $\mathrm{C}^{18} \mathrm{O}(3-2)$ & 0.22 & 5.3 & $1.21 \pm 0.04$ & $0.36 \times 0.31(-51)$ & $22 \times 19$ \\
\hline & ${ }^{13} \mathrm{CO}(3-2)$ & 0.22 & 4 & $4.61 \pm 0.05$ & $0.36 \times 0.32(-52)$ & $22 \times 19$ \\
\hline HD 163296 & $\mathrm{C}^{18} \mathrm{O}(2-1)$ & 0.2 & 2.86 & $7.3 \pm 0.1$ & $0.29 \times 0.20(-88)$ & $29 \times 20$ \\
\hline IM Lup & $\mathrm{C}^{18} \mathrm{O}(2-1)$ & 0.2 & 4 & $1.29 \pm 0.04$ & $0.58 \times 0.39(-76)$ & $93 \times 62$ \\
\hline
\end{tabular}

gas masses and $M_{\star}$, in contrast to a clear correlation between dust mass and $M_{\star}$ (Miotello et al. 2017). These results suggest that $\mathrm{CO}$ depletion, if not the only reason for the weak $\mathrm{CO}$ emission, may occur widely in disks. Its cause is likely a fundamental process in disk evolution and planet formation.

Various chemical and physical processes have been proposed to explain $\mathrm{CO}$ depletion observed in protoplanetary disks. Chemical processes can reprocess $\mathrm{CO}$ into other molecules and gradually reduce the $\mathrm{CO}$ abundance in disks (Aikawa \& Herbst 1999; Bergin et al. 2014; Reboussin et al. 2015; Eistrup et al. 2016; Yu et al. 2016; Bosman et al. 2018; Dodson-Robinson et al. 2018; Schwarz et al. 2018). On the other hand, warm $\mathrm{CO}$ gas diffuses into a colder region closer to the midplane, freezes on dust grains, and is consequently sequestered in the midplane as dust grains grow and settle (Krijt et al. 2016; Xu et al. 2017). As grains radially drift inward, they further redistribute condensed volatiles in the disk (Öberg \& Bergin 2016; Stammler et al. 2017; Krijt et al. 2018). Even starting with a constant $\mathrm{CO}$ abundance in the disk, these chemical and physical processes will lead to a varying $\mathrm{CO}$ gas abundance in the midplane and the warm molecular layer of the disk (Krijt et al. 2018).

However, previous observational studies mostly focused on disk-averaged $\mathrm{CO}$ abundance, and very few studies compared the predictions of depletion mechanisms directly with spatially resolved observations. Here we make the first attempt to investigate whether the $\mathrm{CO}$ depletion varies with radius. Our approach is to compare spatially resolved $\mathrm{CO}$ isotopologue line observations with detailed thermo-chemical models for four well-studied disks. We mainly use $\mathrm{C}^{18} \mathrm{O}$ line observations, as ${ }^{12} \mathrm{CO}$ and ${ }^{13} \mathrm{CO}$ lines are expected to be highly optically thick in most regions of the disk and $\mathrm{C}^{18} \mathrm{O}$ low $J$ lines are mostly optically thin beyond $\sim 30$ au.

This paper is structured as follows. In Section 2, we present the observations of line images and describe the data calibration processes. Section 3 explains the detailed chemical modeling and choices of input parameters. In Section 4, we compare the chemical models with observations and derive $\mathrm{CO}$ gas abundance distributions needed to match with observations. In Section 5, we discuss how the current models of chemical processing and dust evolution in disks may produce the radial
$\mathrm{CO}$ abundance profiles derived here. Then we summarize the findings in Section 6.

\section{Observations}

We employed a sample of four protoplanetary disks with high angular resolution $\mathrm{C}^{18} \mathrm{O}$ line observations from the ALMA archive. The four disks are DM Tau, TW Hya, HD 163296, and IM Lup. These sources were selected based on availability and quality of their $\mathrm{C}^{18} \mathrm{O}$ line observations. The spatial resolution of these observations is between $0 . " 2$ and 0.15 , corresponding to 20-80 au resolution based on distances from Gaia measurements (Gaia Collaboration et al. 2016, 2018). For the TW Hya disk, we included additional ${ }^{13} \mathrm{CO}(3-2)$ line observations to constrain the $\mathrm{CO}$ abundance structure in a $>100$ au region, where its $\mathrm{C}^{18} \mathrm{O}$ (3-2) emission drops below the noise level. The usage of ${ }^{13} \mathrm{CO}$ line for TW Hya is based on previous studies, which showed that the ${ }^{13} \mathrm{CO}(3-2)$ line of the TW Hya disk is optically thin beyond 70 au (Schwarz et al. 2016). We did not include ${ }^{13} \mathrm{CO}$ line observations for the other three disks, because previous studies suggested that ${ }^{13} \mathrm{CO}$ low $J$ lines of these disks are likely to be highly optically thick at most of the disk regions (Williams \& Best 2014; Flaherty et al. 2015; Cleeves et al. 2016). A journal of the ALMA observations is provided in Table 1.

All raw data were calibrated through the accompanying reduction scripts under the corresponding version of CASA (McMullin et al. 2007). Bandpass and phase calibration used bright quasars as calibrators, and the flux calibrations were based on either a solar system object or bright quasars. The absolute flux uncertainty is estimated to be $10 \%$.

The following imaging processes were all performed with CASA 5.0. We carried out self-cal for each data set using continuum visibilities combined from line-free channels. For IM Lup, two data sets from different programs were combined together after individual self-cal, using fixvis and fixplanets tasks in CASA to shift the data sets to a common phase center. The calibrated data were imaged in CASA using the tclean task. Briggs weighting (robust $=0.5$ or 1 ) and the multiscale option with a factor of $(0,5,10)$ were used. The channel widths used for images are 0.2 or $0.22 \mathrm{~km} \mathrm{~s}^{-1}$ (see Table 2), similar to 


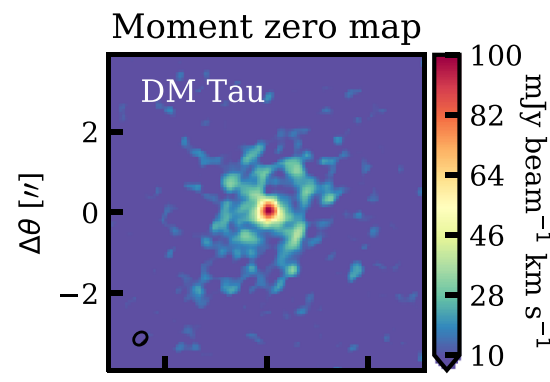

Normalized surface brightness distribution
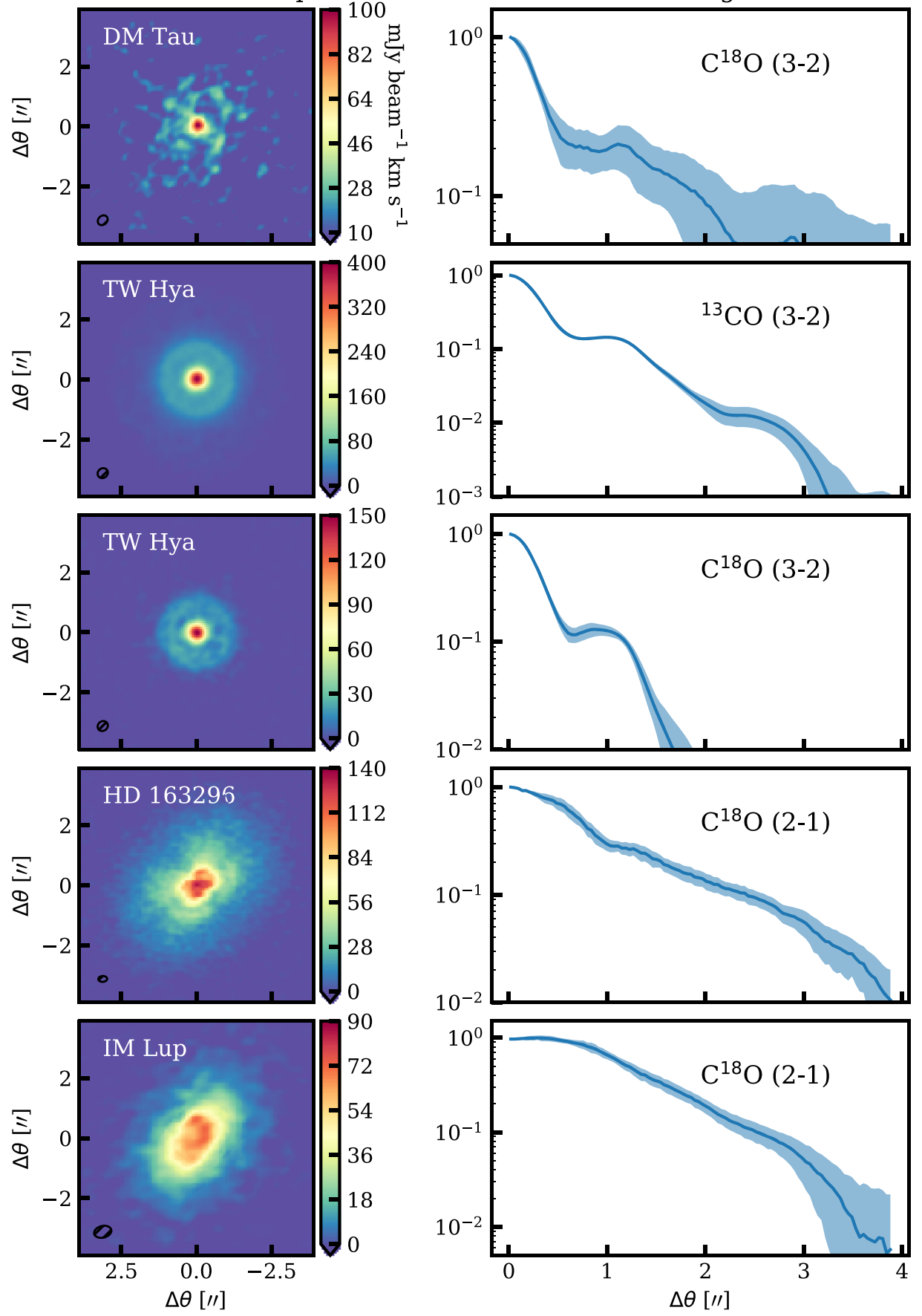

Figure 1. Left column: moment zero map (integrated intensity) of CO isotopologue line observations (continuum subtracted). The axes are labeled with angular offsets from the disk center, and the synthesized beam is shown in the lower left corner of each panel. Right column: deprojected radial line intensity profiles of each line. The shaded areas indicate a $1 \sigma$ uncertainty. The deprojections used the disk geometric parameters listed in Table 3.

the spectral resolution in the original observations, except for HD 163296. Although the original observations of HD 163296 have a higher spectral resolution, we binned the data into a channel width of $0.2 \mathrm{~km} \mathrm{~s}^{-1}$ for a better signal-to-noise ratio. The noise levels and line fluxes of the channel maps can be seen in Table 2. Figure 1 presents moment zero maps of line observations and deprojected radial intensity profiles. The deprojection was based on disk inclination and position angles listed in Table 3.

\section{Methods}

The goal of this study is to investigate how the $\mathrm{CO}$ gas abundance varies with radius in the warm molecular layer of a protoplanetary disk. Our approach is to analyze a sample of well-studied disks in a homogeneous framework, by comparing spatially resolved emission of optically thin $\mathrm{CO}$ isotopologue lines to the predictions of thermo-chemical models of constant carbon elemental abundance across disks. 
Table 3

Source Information

\begin{tabular}{|c|c|c|c|c|c|c|c|c|c|c|c|}
\hline Source & $\begin{array}{c}M_{\star} \\
\left(M_{\odot}\right)\end{array}$ & $\begin{array}{c}R_{\star} \\
\left(R_{\odot}\right)\end{array}$ & $\begin{array}{l}T_{\text {eff }} \\
(\mathrm{K})\end{array}$ & $\begin{array}{c}\mathrm{D} \\
(\mathrm{pc})\end{array}$ & $\begin{array}{c}M_{\mathrm{d}} \\
\left(M_{\odot}\right)\end{array}$ & $\begin{array}{l}M_{\mathrm{gas}} \\
\left(M_{\odot}\right)\end{array}$ & $\begin{array}{c}V_{\mathrm{lsr}} \\
\left(\mathrm{km} \mathrm{s}^{-1}\right)\end{array}$ & $\begin{array}{c}\text { incl } \\
(\mathrm{deg})\end{array}$ & $\begin{array}{l}\text { PA } \\
(\text { deg })\end{array}$ & $\begin{array}{c}L_{X} \\
\left(\mathrm{erg} \mathrm{s}^{-1} \mathrm{~cm}^{-2}\right)\end{array}$ & References \\
\hline DM Tau & 0.53 & 1.25 & 3705 & 140 & $5.00 \mathrm{E}-04$ & $5.00 \mathrm{E}-02$ & 5.95 & 35 & 335 & $3.00 \mathrm{E}+29$ & $1,2,3,4,5$ \\
\hline TW Hya & 0.8 & 1.04 & 4110 & 60 & $5.00 \mathrm{E}-04$ & $5.00 \mathrm{E}-02$ & 2.84 & 7 & 335 & $1.60 \mathrm{E}+30$ & $2,6,7,8$ \\
\hline IM Lup & 1 & 2.5 & 3900 & 160 & $1.70 \mathrm{E}-03$ & $1.70 \mathrm{E}-01$ & 4.5 & 48 & 144 & $4.30 \mathrm{E}+30$ & $2,13,14,15$ \\
\hline
\end{tabular}

Note. (1) Kenyon \& Hartmann (1995); (2) Gaia Collaboration et al. (2018); (3) Andrews et al. (2011); (4) Bergin et al. (2016); (5) Henning et al. (2010); (6) Andrews et al. (2012); (7) Huang et al. (2018a); (8) Brickhouse et al. (2010); (9) Natta et al. (2001); (10) Isella et al. (2016); (11) Rosenfeld et al. (2013a); (12) Swartz et al. (2005); (13) Pinte et al. (2008); (14) Cleeves et al. (2016); (15) Günther et al. (2010); (16) de Gregorio-Monsalvo et al. (2013).

In the following sections, we describe our parameterization on the density structures in protoplanetary disks, assumptions on the input parameters and previous constraints, the numerical methods for radiative transfer and chemical evolution, and our approach for comparing models with observations.

\subsection{Parametric Density Model}

We employ a global surface density from the self-similar solution of a viscously evolving disk (Lynden-Bell \& Pringle 1974), see Equation (1). This profile has been widely used in modeling of protoplanetary disks (e.g., Andrews et al. 2011; Zhang et al. 2014; Cleeves et al. 2016).

$$
\Sigma(R)=\Sigma_{c}\left(\frac{R}{R_{c}}\right)^{-\gamma} \exp \left[-\left(\frac{R}{R_{c}}\right)^{2-\gamma}\right],
$$

where $\Sigma_{c}$ is the surface density at the characteristic radius $R_{c}$, and $\gamma$ is the gas surface density exponent.

The disk model is composed of three mass componentsgas, a small dust population, and a large dust grain population. The separation of different dust populations is used to mimic the effects of dust evolution in protoplanetary disks, including vertical settling and radial drifting (Birnstiel et al. 2012; Krijt et al. 2016). The mass distribution of all three components are assumed to follow the global surface density profile as Equation (1), but each component can have a different set of parameter values. The gas and the small grain population are assumed to be spatially coupled, while the large grain population has a different spatial distribution.

For each mass component, the vertical distribution is characterized by a scale height $h(R)$ and the density $\rho$ is computed through Equation (2)

$$
\begin{gathered}
\rho_{i}(R, Z)=f_{i} \frac{\Sigma(R)}{\sqrt{2 \pi} h_{i}(R)} \exp \left[-\left(\frac{Z}{h_{i}(R)}\right)^{2}\right], \\
h_{i}(R)=\chi_{i} h_{0}(R / 100 \mathrm{au})^{\psi},
\end{gathered}
$$

where $f_{i}$ is the mass fraction of each mass component, $h_{0}$ is the scale height at $100 \mathrm{au}, \psi$ is a parameter that characterizes the radial dependence of the scale height. The large grain population is expected to be more settled compared to the gas and small grains. This is modeled by the settling parameter $\chi_{i}$, where $\chi=1$ of the gas and the small grain population, and $\chi<1$ for the large grain population.

Both dust populations are assumed to follow an MRN size distribution $n(a) \propto a^{-3.5}$ with a minimum grain size $a_{\min }=0.005 \mu \mathrm{m}$, and $a_{\max }=1 \mu \mathrm{m}$ for the small grain population and $a_{\max }=1 \mathrm{~mm}$ for the large grain population. We use
Mie theory to compute the wavelength dependence of dust opacity.

\subsection{The Adoption of Input Parameters and Previous Observational Constraints}

\subsubsection{Disk Parameters}

All four disks are well-studied disks and their disk properties have been under intensive study in the literature. For our purpose of studying $\mathrm{CO}$ abundance structure, the most important parameters are the gas surface density profile and the disk flaring level. The former sets the baseline of $\mathrm{CO}$ gas distribution, and the latter largely determines the temperature structure in a disk. When gas/small dust distribution constraints are available from scattered light images, we adopt these values from previous studies. If not, we adopt $\gamma_{\mathrm{g}}=1$ for gas and small dust grains. The gas disk sizes are adopted from scattered light images or previous studies of multiple $\mathrm{CO}$ isotopologue lines. We vary the $\gamma$ index of gas surface density profiles to test the uncertainties in the derived $\mathrm{CO}$ abundance structures in Section 4.

For a given surface density profile, the flaring level of the disk can be constrained by fitting its broadband spectral energy distribution (SED; Dullemond \& Dominik 2004). The mass ratio of the small-to-large grains and the settling of large grains can affect the thermal structure and the UV penetration in a disk. Schwarz et al. $(2018,2019)$ had carried out systematic tests on the effect of small-to-large dust mass ratio on the $\mathrm{CO}$ processing chemistry. They showed that the $\mathrm{CO}$ depletion happened faster in models with higher small-to-large dust mass ratio. Here we adopt an intermediate level of $80 \%$ of the mass in large grains for modeling. The only exception is the IM Lup disk for which we adopt $99 \%$ of the mass in large grains as this value was used on the comprehensive model of the IM Lup disk of Cleeves et al. (2016). Here we use a settling parameter of $\chi=0.2$ for the large grain population. The detailed disk structures inside 20 au is not critical for our analysis as we focus on $\mathrm{CO}$ gas distribution on a scale of tens of astronomical units. We also ignore small-scale substructures in these disks (Andrews et al. 2016, 2018; Zhang et al. 2016), as this work focuses on the large-scale radial variations. Please see Section 5.2 for a more detailed discussion on possible effects of substructures on $\mathrm{CO}$ abundance distribution.

Two of these four disks (TW Hya and DM Tau) have HD (1-0) line observations and previous models suggest that their gas-to-dust mass ratios are close to 100 (Bergin et al. 2013; McClure et al. 2016). For simplicity, we use a gas-to-dust (small+large) mass ratio of 100 for all four disks. The uncertainty in the total gas mass is not crucial for the study here, as we focus on the radial variation patterns. 
Table 4

Disk Model Parameters

\begin{tabular}{|c|c|c|c|c|c|c|}
\hline & DM Tau & TW Hya & HD 163296 & IM Lup & Units & Definition \\
\hline$\gamma_{\mathrm{g}}$ & 1 & 0.75 & 0.8 & 1 & & surface density index of gas \\
\hline$\gamma_{\mathrm{mm}}$ & 1 & 1 & 0.1 & 0.3 & & surface density index of large grain \\
\hline$R_{c}^{g}$ & 270 & 104 & 165 & 100 & $(\mathrm{au})$ & characteristic radius of gas \\
\hline$R_{c}^{\mathrm{mm}}$ & 135 & 60 & 90 & 100 & $(\mathrm{au})$ & characteristic radius of large grain \\
\hline$M_{\mathrm{mm}}$ & 4.00E-04 & $4.00 \mathrm{E}-04$ & $1.20 \mathrm{E}-03$ & $1.70 \mathrm{E}-03$ & $\left(M_{\odot}\right)$ & mass of large grain \\
\hline$M_{\text {small }}$ & $1.00 \mathrm{E}-04$ & $1.00 \mathrm{E}-04$ & $3.00 \mathrm{E}-04$ & $1.70 \mathrm{E}-05$ & $\left(M_{\odot}\right)$ & mass of small grain \\
\hline$\chi_{\mathrm{mm}}$ & 0.2 & 0.2 & 0.2 & 0.2 & & scale fraction of large grain \\
\hline$H_{100}(\mathrm{au})$ & 6 & 6 & 8.5 & 8 & $(\mathrm{au})$ & gas scale height at $100 \mathrm{au}$ \\
\hline$\psi$ & 1.25 & 1.25 & 1.08 & 1.2 & & flaring parameter \\
\hline$R_{i n}^{\mathrm{mm}}$ & 19 & 1 & 0.5 & 0.2 & $(\mathrm{au})$ & inner radius of large grain \\
\hline$R_{\mathrm{in}}^{\mathrm{g}}$ & 3 & 4 & 0.5 & 0.2 & $(\mathrm{au})$ & inner radius of gas/small grain \\
\hline$R_{\text {out }}^{\mathrm{mm}}$ & 300 & 70 & 600 & 313 & $(\mathrm{au})$ & outer radius of large grain \\
\hline$R_{\text {out }}^{\mathrm{g}}$ & 500 & 200 & 600 & 1200 & $(\mathrm{au})$ & outer radius of gas/small grain \\
\hline
\end{tabular}

Table 4 lists all the parameters adopted for our disk models. Here we briefly describe the adoption of parameters for each disk:

1. DM Tau: we employ a $\gamma_{\mathrm{g}}=1$ for gas and small grains, as no scattered light image studies are available. The inner edge of gas is set to be 4 au (Calvet et al. 2005). The characteristic radius of gas, $R_{c}^{\mathrm{g}}$, is set to $270 \mathrm{au}$ based on the extent of $\mathrm{C}^{18} \mathrm{O}(3-2)$ emission in channels close to the stellar velocity. For the large grain population, we use $\gamma_{\mathrm{mm}}=1, R_{c}^{\mathrm{mm}}=135 \mathrm{au}$, and an inner radius of $20 \mathrm{au}$ based on the model of Andrews et al. (2011), which matches the $880 \mu \mathrm{m}$ continuum observations of DM Tau.

2. TW Hya: we adopt the general gas surface density profile of TW Hya derived from scattered light images from van Boekel et al. (2017). This distribution has a global $\gamma_{\mathrm{g}}=0.7$ and a exponential taper beyond $104 \mathrm{au}$. The distribution of millimeter-grain is truncated at $70 \mathrm{au}$, with a $R_{c}^{\mathrm{mm}}=60$ au (Andrews et al. 2012).

3. HD 163296: we employ the basic structure of Isella et al. (2016), which fitted the $1.3 \mathrm{~mm}$ continuum emission and the general sizes of $\mathrm{CO} /{ }^{13} \mathrm{CO} / \mathrm{C}^{18} \mathrm{O}(2-1)$ isotopologue emissions. The model uses $R_{c}^{\mathrm{g}}=165$ au and $\gamma_{\mathrm{g}}=0.8$ for gas and small grains, and $R_{c}^{\mathrm{mm}}=90 \mathrm{au}, \gamma_{\mathrm{mm}}=0.1$ for the large grain population.

4. IM Lup: we adopt $\gamma_{\mathrm{g}}=1$ for gas, and other surface density profile parameters from Cleeves et al. (2016), which carried out a comprehensive model of $875 \mu \mathrm{m}$ continuum and multiple CO (2-1) isotopologue emissions.

\subsubsection{Stellar Parameters}

We adopt the effective temperatures and luminosities of the central stars from the literature and rescale the stellar luminosities using the latest Gaia 2 measurements. The inclination and position angles are adopted from previous analysis of spatially resolved line and continuum observations. The UV and X-ray luminosities are from the literature. All stellar information and disk geometric parameters are listed in Table 3.

\subsection{The Numerical Methods}

The general modeling processes are outlined in Figure 2.

We first use the radiative transfer code RADMC3D to calculate the dust thermal structure in a disk (Dullemond et al. 2012) and then generate model SEDs to compare with observations. To constrain the vertical scale height of dust, we first run a grid of $h_{0}$ and $\psi$ to search for best-fitting SED models of each disk. The $h_{0}$ range is from 0.02 to 0.2 with a step size of 0.02 , and $\phi$ from 1 to 1.3 with a step size of 0.05 . The best-fitting SED models are shown in Figure 3.

Then we adopt the best-fitting values of density structure into a full 2D time-dependent thermo-chemical code RAC2D. This code computes the disk chemistry and the heating-cooling balance for both the gas and the dust self-consistently. We briefly describe the code here and interested readers can find a full description in Du \& Bergin (2014). Given a static density structure of gas and dust, the code first solves the dust temperature and radiation field (from X-ray to centimeter wavelengths) in the disk using a Monte Carlo approach. The cosmic-ray ionization inside the disk is simulated with an attenuation length of $96 \mathrm{~g} \mathrm{~cm}^{-2}$ (Umebayashi \& Nakano 1981; Bergin et al. 2007). For the given radiation field, the code then simultaneously solves the chemical evolution and gas thermal structure in the disk. The chemical network has 467 species and 4801 reactions, including the full gas-phase network from the UMIST database, dissociation of $\mathrm{H}_{2} \mathrm{O}$ and $\mathrm{OH}$ by Ly $\alpha$ photons, adsorption and desorption of species on the dust grain surface either thermally or induced by cosmic rays and UV photons, and two-body reactions on the dust grain surface.

The chemical models are initialized with a composition listed in Table 1 of $\mathrm{Du} \&$ Bergin (2014). In particular, all carbon is initially in $\mathrm{CO}$ gas with an abundance of $1.4 \times 10^{-4}$ relative to $\mathrm{H}$ atoms and a binding energy of $855 \mathrm{~K}$ for $\mathrm{CO}$ ice (Öberg et al. 2005). We let the chemistry run for $1 \mathrm{Myr}$ for all models. No isotopologue fractionation is considered in the chemical network, as the fractionation is expected to be insignificant in massive disks like this sample (Miotello et al. 2014). We rescale the output $\mathrm{CO}$ abundance by the local ISM ratios of $\mathrm{CO} /{ }^{13} \mathrm{CO}=69$, and $\mathrm{CO} / \mathrm{C}^{18} \mathrm{O}=570$ to generate $\mathrm{CO}$ isotopologue abundances (Wilson 1999).

Using the gas temperature and $\mathrm{CO}$ abundance structures, we compute line images using the ray-tracing module of RAC2D, assuming an LTE condition. We initially simulate the images at a 2 au spatial resolution and $10 \times$ spectral resolution as the observations, and then convolve model images to the same spatial and spectral resolution as observations. 


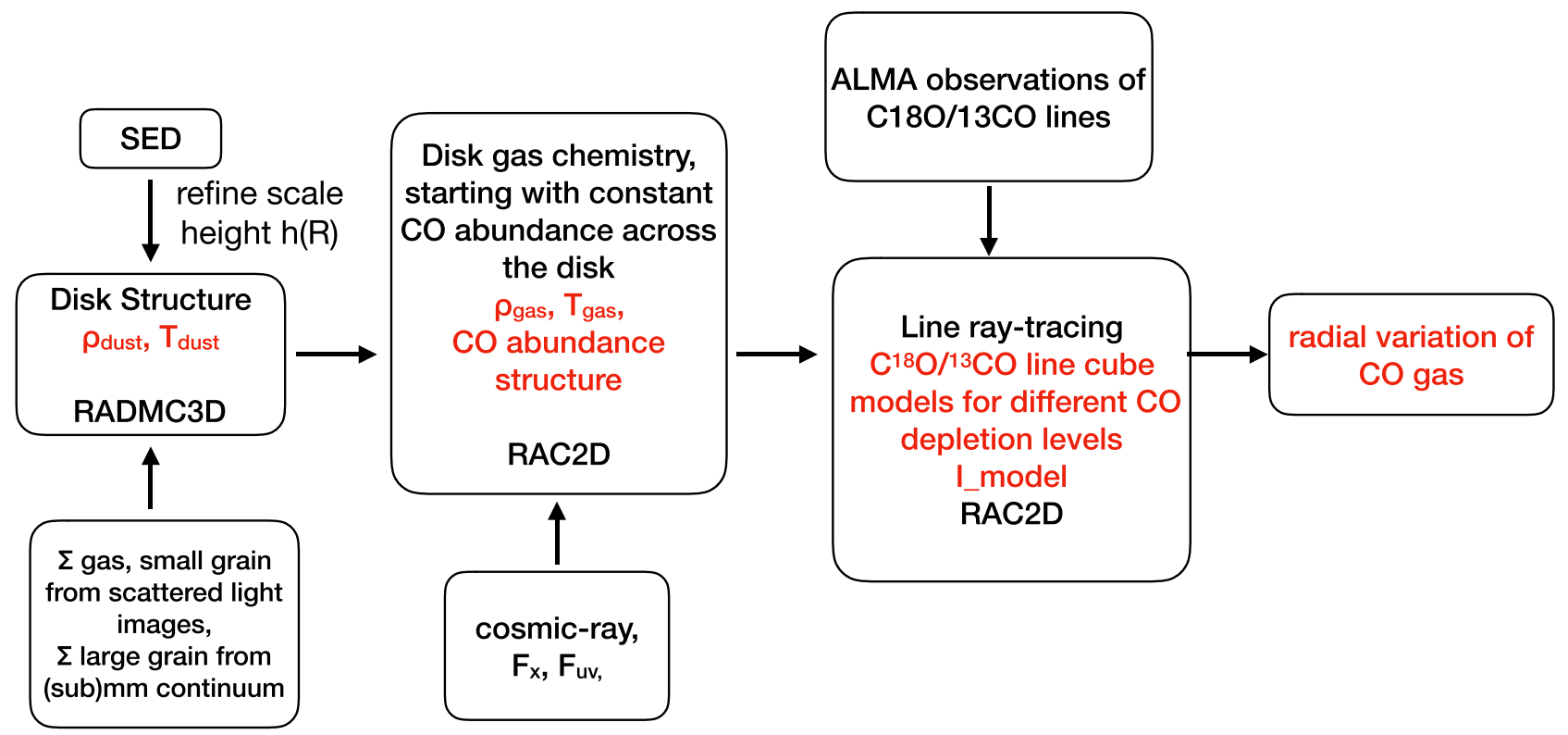

Figure 2. An outline of the modeling processes. The output of each step is highlighted in red.
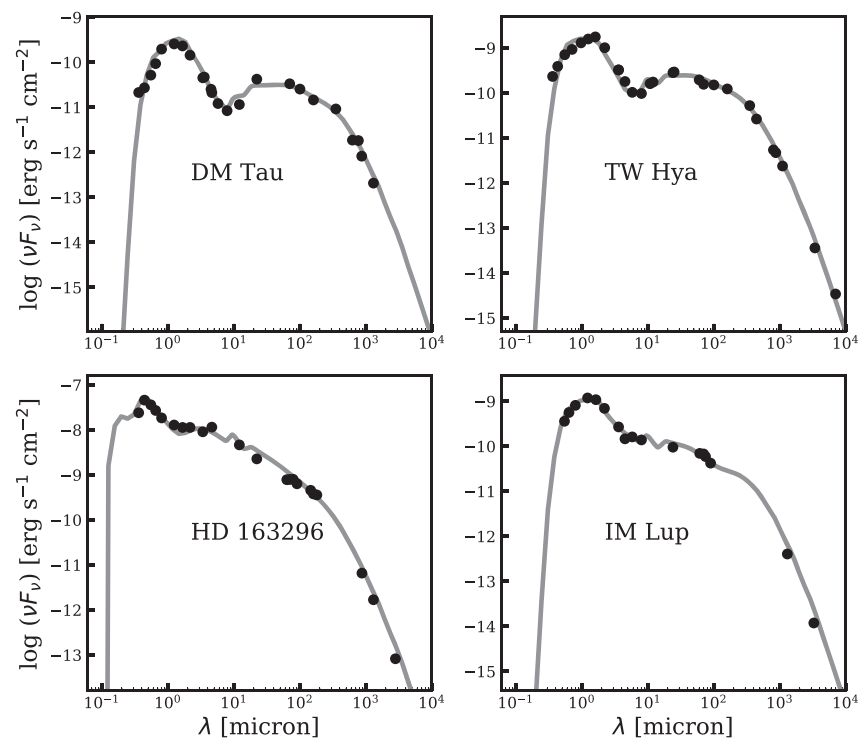

Figure 3. The best-fitting SED models of the four disks. The photometry data are black dots and the best-fitting models are overlaid as solid gray lines.

\subsection{Parameter Tests}

We test model uncertainties of two important parameters: the cosmic-ray ionization rate $\left(\zeta_{\mathrm{CR}}\right)$ and the $\gamma$ index for surface density profiles.

Previous chemical studies suggested that an ISM level of cosmic-ray $(\mathrm{CR})$ ionization rate $\left(\zeta_{\mathrm{CR}} \geqslant 1.36 \times 10^{-17} \mathrm{~s}^{-1}\right)$ is critical for sufficient $\mathrm{CO}$ processing to other molecules (Bosman et al. 2018; Schwarz et al. 2018). This level of cosmic-ray rate is typical in ISM but it is unclear whether this is also present in disks, as disk wind or magnetic fields in actively accreting $\mathrm{T}$ Tauri disk systems may attenuate the cosmic-ray rate in disks (Cleeves et al. 2013, 2015). To test the impact of CR rate on our chemical models, we run two sets of models: a set of standard models with a $\zeta_{\mathrm{CR}}=1.36 \times 10^{-18} \mathrm{~s}^{-1}$ and the other set with a high CR rate of $1.36 \times 10^{-17} \mathrm{~s}^{-1}$.
We also test how uncertainties on the slope of surface density profiles would impact the constraints on $\mathrm{CO}$ depletion profile. Therefore, for each disk, we run two additional models with $\gamma$ varies by \pm 0.2 . The characteristic radii and total gas masses are kept the same.

\section{Results}

\subsection{CO Abundance and Gas Temperature Structures from Thermo-chemical Models}

In Figure 4, we show the $\mathrm{CO}$ gas abundance and gas temperature structures in the standard models $\left(\zeta_{\mathrm{CR}}=1.36 \times\right.$ $10^{-18} \mathrm{~s}^{-1}$ ) of these four disks. All four disks show a molecular layer in which the $\mathrm{CO}$ gas abundance is close to $10^{-4}$. This layered structure has been seen in channel maps of $\mathrm{CO}$ line observations of HD 163296 and IM Lup (Rosenfeld et al. 2013b; Pinte et al. 2018a). The last column in Figure 4 shows the column density distributions $\mathrm{CO}$ gas and ice in the four disk models. The $\mathrm{CO}$ (gas+ice) column density distribution is close to $10^{-4}$ at all radii, indicating very little $\mathrm{CO}$ has been converted to other carbon carriers after $1 \mathrm{Myr}$ in these low CR rate models.

When no significant conversion of $\mathrm{CO}$ into other carriers occurs, the $\mathrm{CO}$ snowline location is predominantly determined by the thermal structure of the disk. DM Tau is the coldest disk in our sample and its CO midplane snowline is around $10-15$ au in the standard model. In the TW Hya model, the CO midplane snowline is around $20 \mathrm{au}$, consistent with $21 \pm 1.3 \mathrm{au}$ derived from ${ }^{13} \mathrm{C}^{18} \mathrm{O}(3-2)$ line observations (Zhang et al. 2017) and the $\mathrm{N}_{2} \mathrm{H}^{+}$line image (Qi et al. 2013). For the HD 163296 model, its $\mathrm{CO}$ midplane snowline is between 50 and $70 \mathrm{au}$, consistent with the previous estimation from $\mathrm{C}^{18} \mathrm{O}$ and $\mathrm{N}_{2} \mathrm{H}^{+}$line observations, after correcting to the Gaia2 distance (Qi et al. 2015). For the IM Lup model, its $\mathrm{CO}$ ice starts to appear around $30 \mathrm{au}$, but its $\mathrm{CO}$ ice column density increases slowly with radius compared with other disks, because the IM Lup disk is highly flaring and has a thicker $\mathrm{CO}$ gas layer than others.

In short, the four standard models $\left(\zeta_{\mathrm{CR}}=1.36 \times 10^{-18} \mathrm{~s}^{-1}\right)$ all show a layered $\mathrm{CO}$ gas structure and the midplane $\mathrm{CO}$ snowline locations are consistent with existing observational 

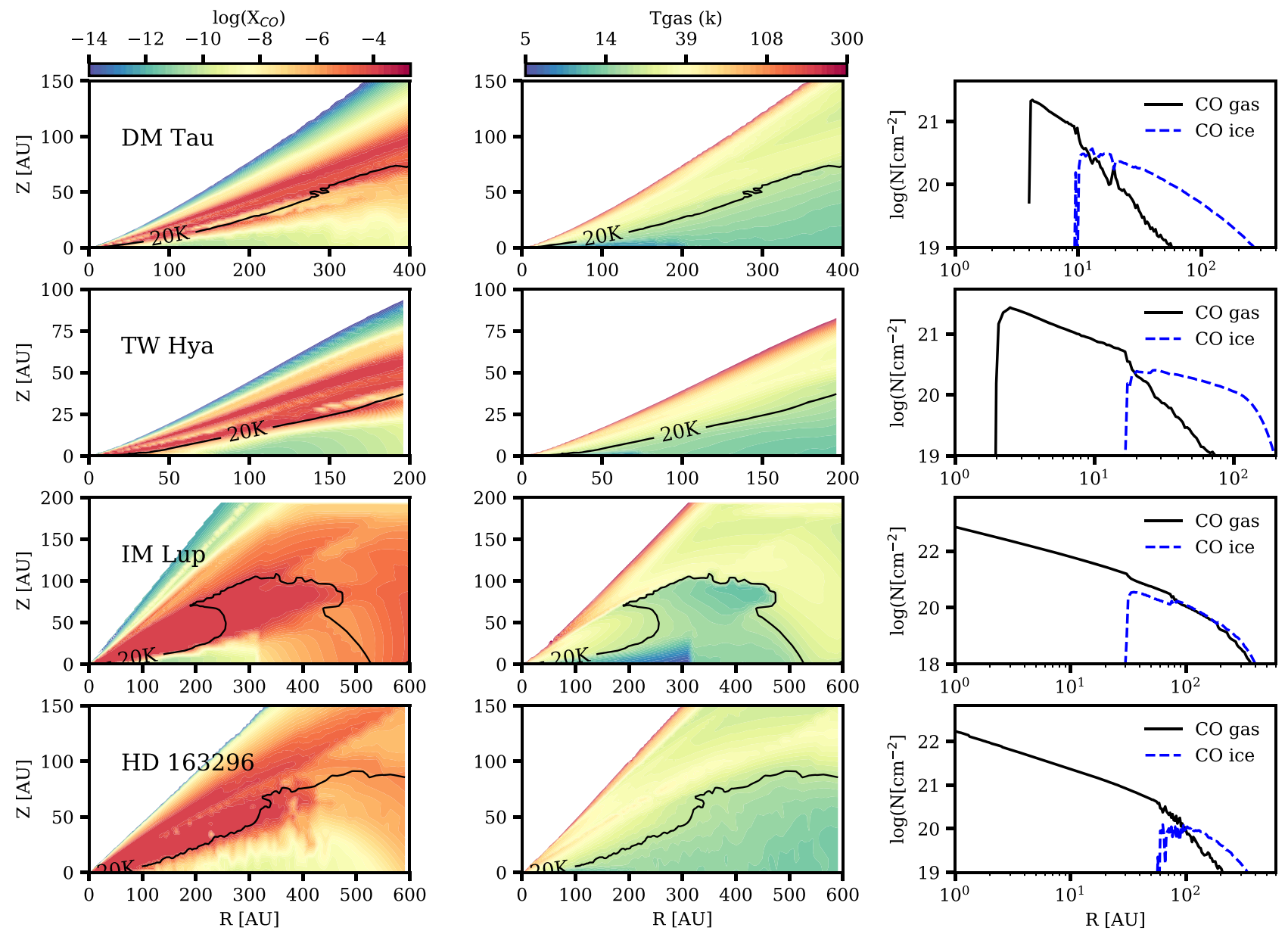

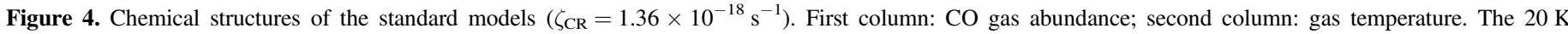

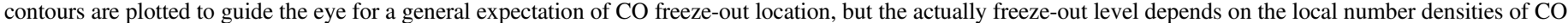

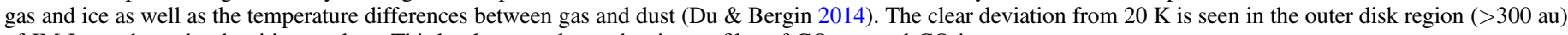
of IM Lup where the densities are low. Third column: column density profiles of CO gas and CO ice.

constraints. In these models, less than $10 \%$ of total CO (gas + ice) have been converted to other carbon carriers after $1 \mathrm{Myr}$.

\subsection{CO Abundance Structures in Models with a High Cosmic- Ray Rate}

Figure 5 shows the comparison of $\mathrm{CO}$ gas column density distribution in high and standard models $\left(\zeta_{\mathrm{CR}}=1.36 \times\right.$ $10^{-17} \mathrm{~s}^{-1}$ versus $1.36 \times 10^{-18} \mathrm{~s}^{-1}$ ). Models of DM Tau and TW Hya show substantial CO gas depletions between 10 and $100 \mathrm{au}$, by a factor of 5-20 after 1 Myr. The HD 163296 model shows an intermediate level of depletion by up to a factor of 10 between 30 and 200 au. The IM Lup model shows the smallest variation between high and low CR rate models with a depletion factor of less than 4 throughout the whole disk.

In general, the chemical processing rates of $\mathrm{CO}$ gas depend on the thermal and ionization structures of disks, which in turn depends on many physical properties, including the stellar luminosity, UV luminosity, disk flaring structure, and dust size distribution. For a given cosmic-ray rate, the available surface areas on grain surface have a significant impact on the $\mathrm{CO}$ processing rate. Schwarz et al. (2018) and Bosman et al. (2018) have carried out systematic studies on how disk properties would impact the CO depletion level, including small/large grain mass ratio and temperature. In our models, there are some very noticeable effects. The effect of grain surface area is most obvious in the IM Lup disk models that have only $1 \%$ of the solid mass in small grains. Limited by the available grain surface areas, the chemical processing of CO in the IM Lup models is order(s) of magnitude smaller than that of other disks. The effect of thermal structure can be seen in HD 163296 models. As a Herbig disk, its gas temperature is much warmer than $\mathrm{T}$ Tauri disks and thus has less $\mathrm{CO}$ freeze-out regions for $\mathrm{CO}$ to be processed in the grain surface path.

Despite significant decreases in the $\mathrm{CO}$ gas column density, Figure 5 shows that the surface brightness profiles of $\mathrm{C}^{18} \mathrm{O}$ line emission are not very sensitive to the $\mathrm{CO}$ depletion caused by high CR ionization. This is because most of the depletion occurs in the deep region of these disks and the $\mathrm{CO}$ emission above the depletion region is already optically thick. These results indicate that the $\mathrm{CO}$ depletion by chemical processing alone, even by high CR rate, is unlikely to account for the low surface brightness observed in these four disks if their gas-todust ratio is still close to 100 .

\subsection{How the CO Surface Brightness Profile Changes with Gas Mass Distribution}

Figure 6 shows how the profiles of total gas surface column density $\left(\Sigma_{\mathrm{g}}\right)$, $\mathrm{CO}$ gas column density $\left(\Sigma_{\mathrm{CO}}\right)$, and the surface 

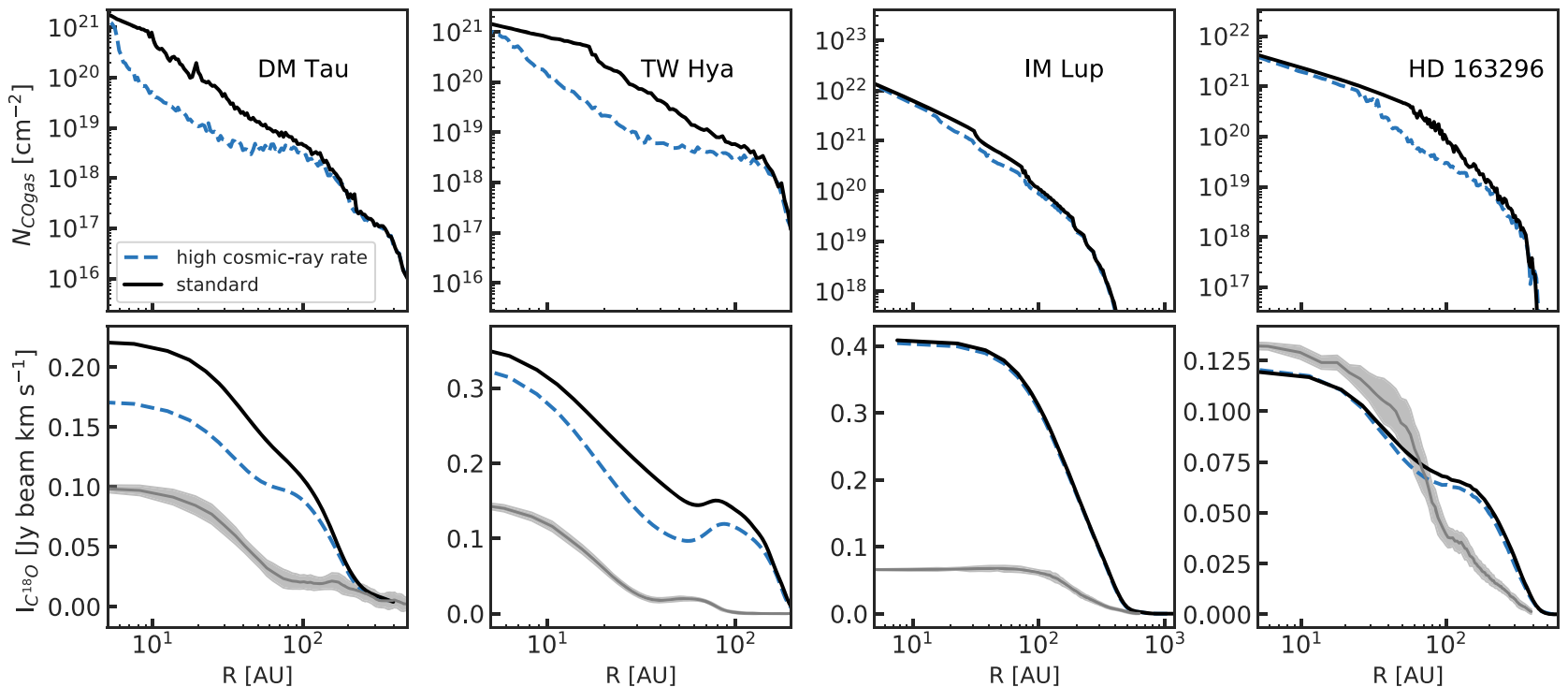

Figure 5. Comparison between high cosmic-ray ionization models and standard models $\left(\zeta_{\mathrm{CR}}=1.36 \times 10^{-17} \mathrm{~s}^{-1}\right.$ vs. $\left.1.36 \times 10^{-18} \mathrm{~s}^{-1}\right)$. Top: CO gas column density in high $\zeta_{\mathrm{CR}}$ (blue dash line) and standard models (black solid line). Bottom: $\mathrm{C}^{18} \mathrm{O}$ line surface brightness profiles in the models with different $\zeta_{\mathrm{CR}}$ rates (blue dash and black solids lines). The observed profiles are in gray.
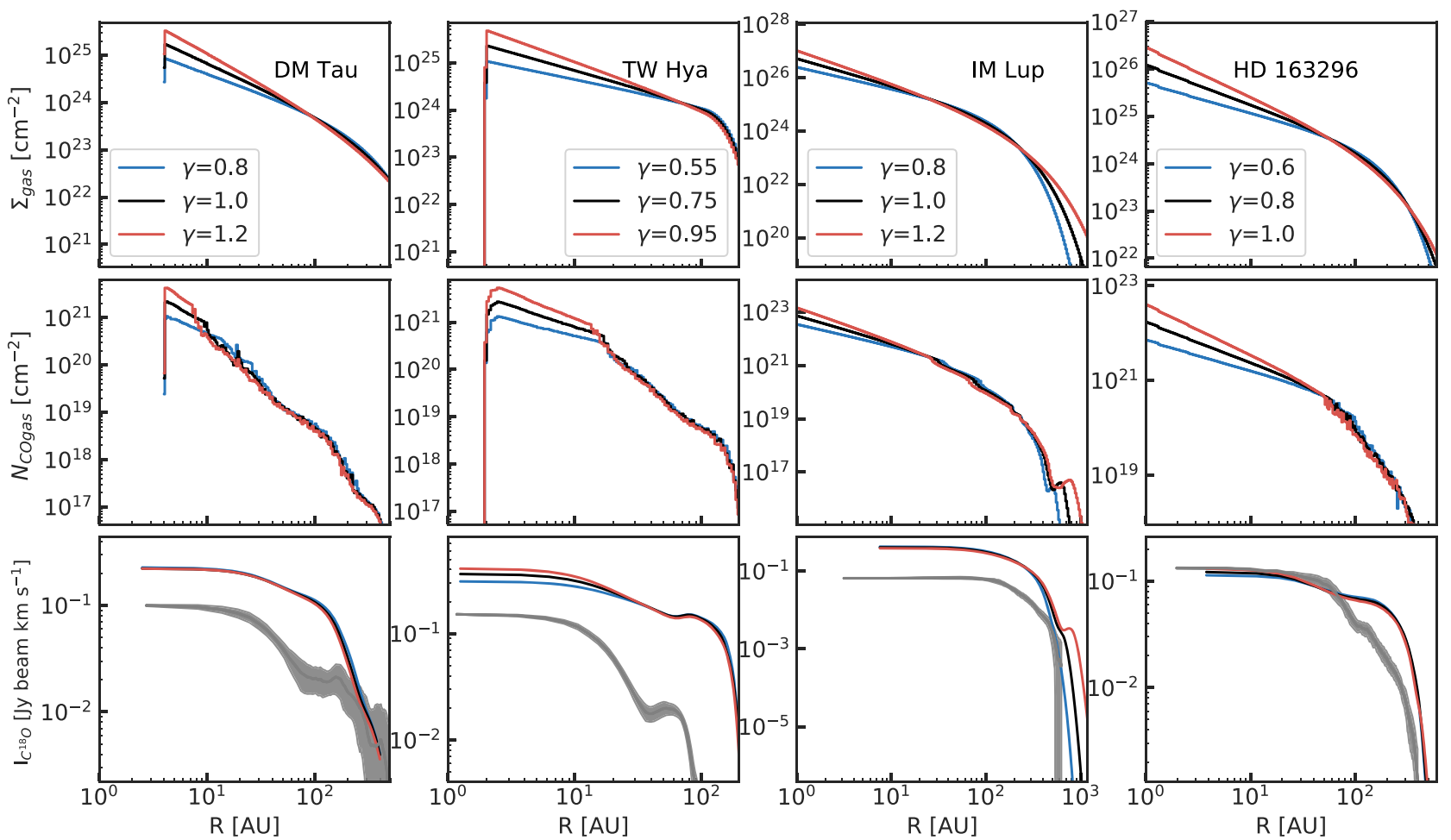

Figure 6. Top row: the total gas surface density profiles for models with different $\gamma$. Middle row: the $\mathrm{CO}$ gas column density profiles after $1 \mathrm{Myr}$ chemical evolution and a $\zeta_{\mathrm{CR}}$ of $1.36 \times 10^{-18} \mathrm{~s}^{-1}$. Bottom row: model $\mathrm{C}^{18} \mathrm{O}$ line brightness distributions vs. observations (gray line).

brightness of $\mathrm{CO}$ isotopologue line emission $\left(I_{\mathrm{CO}}\right)$ vary in models deviating from the $\gamma$ of standard models by \pm 0.2 . These results show that $\Sigma_{\mathrm{CO}}$ represents well the gas column density change within the midplane $\mathrm{CO}$ snowline, but it becomes less sensitive to the gas surface density changes beyond the $\mathrm{CO}$ snowline as the $\mathrm{CO}$ gas layer only accounts for a small fraction of the total gas column. The $I_{\mathrm{CO}}$ profiles only change less than $30 \%$ for uncertainty of 0.2 in the $\gamma$ index.

\subsection{Radial Distribution of CO Gas abundance: Models versus Observations}

Here we compare the surface brightness distributions of $\mathrm{CO}$ lines $\left(I_{\mathrm{CO}}\right)$ between observations and our standard models to estimate the levels of variations in the radial $\mathrm{CO}$ gas abundance. For each disk, we generate a set of synthetic line images by rescaling the model $\mathrm{CO}$ gas abundance throughout the disk with a constant factor. The grid spans from a depletion factor of $0.1-400$, increasing by a factor of 1.2 at each step. 

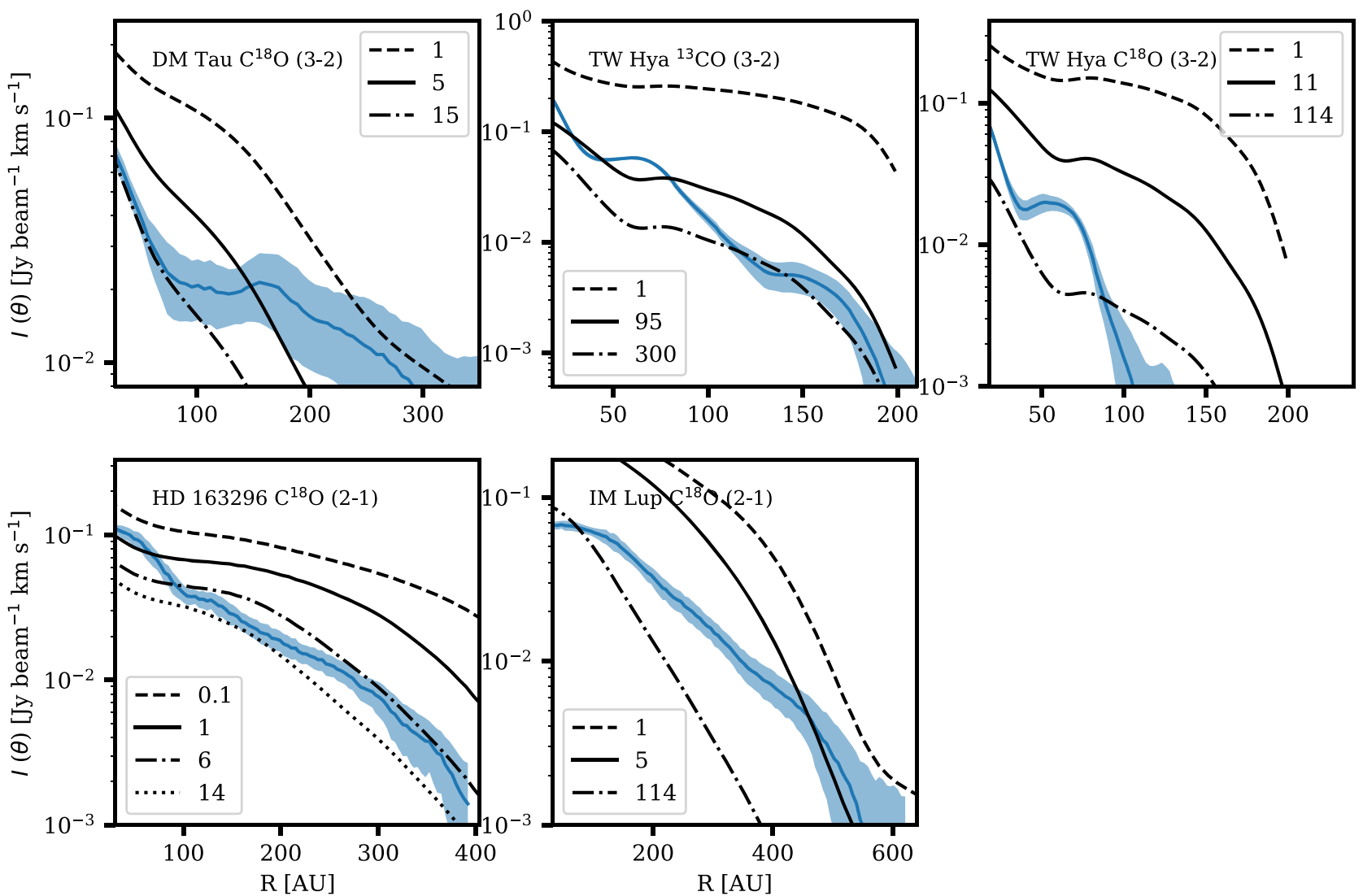

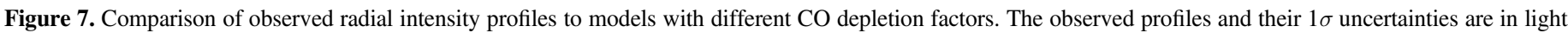
blue and the models are in black.

Figure 7 shows the line surface brightness profiles of models with exemplary depletion scales, comparing with the observed profiles in the four disks. Using the grid of models, we record which depletion factor matches the best with models at each radius.

Figure 8 shows the required $\mathrm{CO}$ depletion distributions to match observations. On the disk-averaged scale, we find that significant depletion of $\mathrm{CO}$ gas abundance is needed to explain observations. This is consistent with the previous results of disk-averaged CO abundance studies (Favre et al. 2013; Flaherty et al. 2015; Cleeves et al. 2016; McClure et al. 2016). For the radial variation in individual disks, we find that one order of magnitude radial variation is usually needed to account for observations. Comparing to the changes of $I_{\mathrm{CO}}$ due to $\gamma$ uncertainty, it is unlikely these large deviations can be explained by surface density profiles alone.

For individual disks, the DM Tau disk shows a nearly constant level of CO depletion inside 100 au (by a factor of 12) and its CO abundance gradually increases with radius to an interstellar ratio at $280 \mathrm{au}$. TW Hya shows a more complicated pattern, with a sharp decrease of $\mathrm{CO}$ abundance between 10 and $30 \mathrm{au}$, and then increases with the radius until $60 \mathrm{au}$, and outside $100 \mathrm{au}$ the CO abundance is extremely depleted ( $>$ a factor of 200). IM Lup shows a monotonic trend of $\mathrm{CO}$ abundance depletion, with an outer disk that has less depletion. HD 163296 shows a modest CO abundance enhancement inside 70 au (by a factor of 2-5), its 100-200 au shows the largest depletion and then a shallow increase of $\mathrm{CO}$ abundance with radius.
Except for the oldest disks TW Hya, the other three disks show a similar pattern in their $\mathrm{CO}$ abundance structures in regions beyond their $\mathrm{CO}$ snowline-the $\mathrm{CO}$ gas abundance is low in the intermediate disk region outside the midplane $\mathrm{CO}$ snowlines, but gradually increases with radius to a higher value in the outermost region. Interestingly, this pattern is consistent with the general trends of carbon elemental abundance suggested by other carbon carriers. Bergner et al. (2019) analyzed $\mathrm{C}_{2} \mathrm{H}$ radial brightness distributions in the same three disks and their models required a high depletion of elemental carbon abundance (by a factor of 10-100) between 100-200 au and only a modest depletion beyond 200 au (by a factor of 1-10). Therefore, the CO depletion profiles likely represent a general depletion of carbon elemental abundance in the atmospheres of these disks.

Summary of model results: (1) In detailed thermo-chemical models for the four disks, a high $\mathrm{CR}$ ionization rate can significantly reduce the CO gas column between 50-100 au up to a factor of 20 after $1 \mathrm{Myr}$. However, the depletions by chemical processing mostly happen at the deep interior of these model disks, and the resulting $\mathrm{CO}$ abundance structures cannot reproduce the weak $\mathrm{CO}$ isotopologue lines observed. (2) Comparing these thermo-chemical models to spatially resolved observations, we find that the $\mathrm{CO}$ gas abundance likely varies significantly with radius. An interesting pattern in the radial profiles is that the $\mathrm{CO}$ gas abundance is low in the intermediate disk region outside the midplane $\mathrm{CO}$ snowlines, but gradually increases with radius to a higher value in the outermost region. 


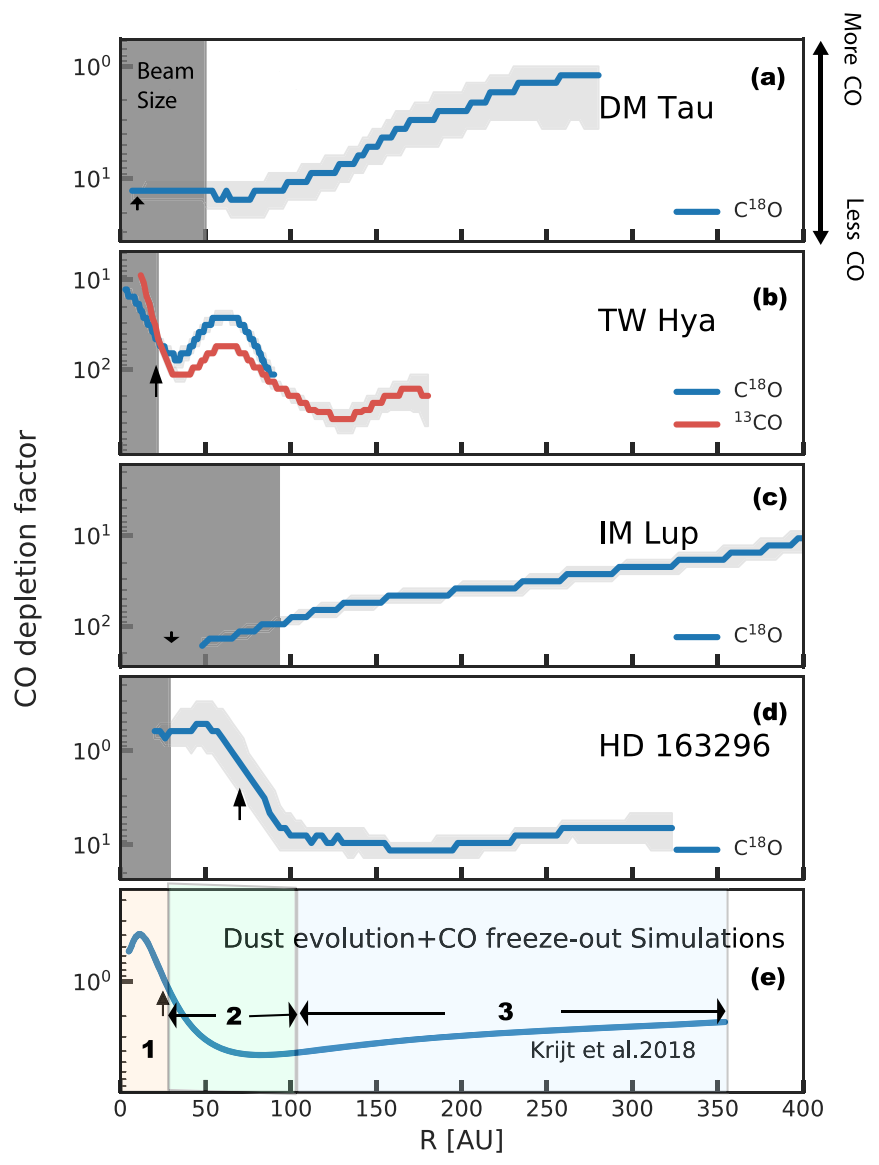

Figure 8. (a)-(d) The radial profiles of $\mathrm{CO}$ depletion factor in four disks. The black arrow indicates the location of $\mathrm{CO}$ snowline at the disk midplane and the gray shaded areas indicate the beam size of the observations. (e) The radial profile of $\mathrm{CO}$ depletion of the warm molecular layer in the 2D dust evolution simulations of Krijt et al. (2018). The transparent colored boxes indicate three regions with different $\mathrm{CO}$ gas abundances: (1) the enhancement of $\mathrm{CO}$ gas abundance inside the midplane $\mathrm{CO}$ snowline, (2) the largest $\mathrm{CO}$ depletion region, and (3) the $\mathrm{CO}$ gas abundance slowly increases with radius.

\section{Discussion}

\subsection{CO Depletion Mechanisms}

Current surveys of nearby star formation regions suggest that gas mass derived from $\mathrm{CO}$ observations is 1-2 orders of magnitude too low compared to the canonic CO ratio in ISM (Ansdell et al. 2016; Long et al. 2017; Miotello et al. 2017). In a broad sense, two types of depletion mechanisms have been proposed: (1) the first one is chemical processes that convert $\mathrm{CO}$ into other less volatile molecules (e.g., $\mathrm{CO}_{2}, \mathrm{CH}_{3} \mathrm{OH}, \mathrm{CH}_{4}$; Aikawa et al. 1999; Bergin et al. 2014; Reboussin et al. 2015; Eistrup et al. 2016; Yu et al. 2016; Bosman et al. 2018; Schwarz et al. 2018); (2) the second type is physical processes that $\mathrm{CO}$ gas abundance is altered as a result of the $\mathrm{CO}$ freezeout onto dust grains and the subsequent dust growth, settling, and radial drifting in protoplanetary disks (Kama et al. 2016; Xu et al. 2017; Krijt et al. 2018). In other words, CO becomes trapped as ice in large grains that reside in the midplane or perhaps even in planetesimals. Previous studies of $\mathrm{CO}$ depletion mechanisms were mostly theoretical works. Here we discuss our results of radial $\mathrm{CO}$ variations in the context of these mechanisms proposed.

\subsubsection{Chemical Depletion Mechanisms}

Several chemical pathways have been proposed to account for $\mathrm{CO}$ depletion in protoplanetary disks. In gas-phase reactions, $\mathrm{CO}$ can be destroyed by $\mathrm{He}^{+}$and subsequently form $\mathrm{CH}_{4}$ and hydrocarbons (Aikawa et al. 1999; Eistrup et al. 2016; $\mathrm{Yu}$ et al. 2016; Schwarz et al. 2018). In grain surface reactions, $\mathrm{CO}$ ice can react with $\mathrm{OH}$ to form $\mathrm{CO}_{2}$, or $\mathrm{CO}$ ice goes through a sequence of hydrogenation to form $\mathrm{CH}_{3} \mathrm{OH}$ (Yu et al. 2016; Bosman et al. 2018; Schwarz et al. 2018, 2019).

All of these destruction pathways require dissociation or ionization radiation, either from UV, X-ray, or cosmic-ray. In general, models suggested that a CR ionization rate larger than $10^{-17} \mathrm{~s}^{-1}$ is needed to make an order of magnitude depletion of $\mathrm{CO}$ gas over a few megayear timescale of the gas lifetime in disks (Bosman et al. 2018; Schwarz et al. 2018). However, it is unclear that a high CR rate is present in actively accreting $\mathrm{T}$ Tauri disks, because of cosmic-ray exclusion by winds and/or strong magnetic fields (Cleeves et al. 2013, 2015).

Previous chemical studies focused on the total gas column density of $\mathrm{CO}$ but did not consider how the vertical location and optical depth would impact the observed $\mathrm{CO}$ line intensities. As we showed in Figure 5, although the $\mathrm{CO}$ gas column density decreases by a factor of 10 between 50 and $100 \mathrm{au}$ in the high CR rate models of the TW Hya and DM Tau disks, the surface brightness of their $\mathrm{C}^{18} \mathrm{O}$ line emission only changes less than $20 \%$, because the $\mathrm{CO}$ processing preferentially occurs at the deep layer while the $\mathrm{CO}$ gas abundance near the surface layer is not significantly depleted.

Therefore, for relatively massive disks $\left(\geqslant 0.01 M_{\odot}\right)$, even when an ISM level CR rate is present and causes an order of magnitude depletion in the total $\mathrm{CO}$ gas column density, the predicted $\mathrm{C}^{18} \mathrm{O}$ lines are still too strong compared with observations.

We will discuss ways to increase $\mathrm{CO}$ depletion at the surface layer in Section 5.1.3.

\subsubsection{Physical Depletion Mechanisms}

Alternatively, the $\mathrm{CO}$ gas depletion may be a result of dust evolution in disks. Detailed models of dust evolutionincluding grain coagulation, fragmentation, and settling combined with viscous gas mixing to deeper layers where grains are growing - have shown the settling and radial drifting of icy pebbles can change the vertical and radial distribution of the CO gas in the disk. Indeed, 1D-models showed that the vertical mixing and dust settling at a single radius can deplete the gas-phase $\mathrm{H}_{2} \mathrm{O}$ and $\mathrm{CO}$ abundance in the warm molecular layer by a factor of a few to 50, depending on the strength of turbulence and the location of the surface $\mathrm{CO}$ snowline (Krijt et al. 2016; $\mathrm{Xu}$ et al. 2017).

Krijt et al. (2018) constructed a 2D model that includes both vertical settling and radial drifting processes to investigate how the $\mathrm{CO}$ gas abundance evolves across the disk. In the vertical direction, they confirmed that within $1 \mathrm{Myr}$, dust evolution can remove up to $80 \%$ of the original $\mathrm{CO}$ vapor in the warm molecular layer outside the CO midplane snowline. In the radial direction, they showed that pebble formation and motion in the disk makes an interesting pattern on the radial distribution of $\mathrm{CO}$ gas abundance in the warm molecular layer (see Figure 8): (1) in regions inside the midplane $\mathrm{CO}$ snowline, $\mathrm{CO}$ gas abundance is enhanced by a factor of a few compared 
to its initial value, (2) the largest $\mathrm{CO}$ gas depletion occurs at an intermediate region beyond the midplane $\mathrm{CO}$ snowline and peaks at a radius around a factor of 2-3 larger than the $\mathrm{CO}$ snowline, (3) in the outermost region beyond the $\mathrm{CO}$ depletion peak, the CO gas abundance smoothly increases with radius.

This radial pattern emerges because the $\mathrm{CO}$ depletion level at a given radius depends on the local timescales of dust growth and vertical mixing, and the supply of $\mathrm{CO}$ from other radial zones. The dust growth and vertical mixing timescales are expected to be proportional to the local orbital timescale (Birnstiel et al. 2012; Krijt et al. 2016). The intermediate region beyond the $\mathrm{CO}$ midplane snowline has shorter timescales compared with that of the outermost region, and therefore its $\mathrm{CO}$ gas depletes faster than that of the outermost region. The region just beyond the midplane $\mathrm{CO}$ snowline does not have the highest depletion because a fraction of $\mathrm{CO}$ vapor inside the snowline can diffuse into this region. The enhancement of $\mathrm{CO}$ gas at the inner disk region occurs when sufficient pebbles with a $\mathrm{CO}$ ice mantle drift inwards into the region inside the $\mathrm{CO}$ snowline and evaporate $\mathrm{CO}$ into the gas phase.

Comparing to our results, the depletion patterns of three disks (except for TW Hya) are qualitatively consistent with this radial pattern due to dust evolution: relatively large $\mathrm{CO}$ depletion beyond the midplane $\mathrm{CO}$ snowline and the outermost disk region is less depleted than the intermediate region. It is interesting that the CO depletion pattern in the HD 163296 disk is strikingly similar to the depletion pattern predicted by Krijt et al. (2018). In the HD 163296 disk, CO gas abundance appears to be enhanced by a factor of 5 inside its midplane snowline. This kind of enhancement is expected if grains with $\mathrm{CO}$ ice radially drift through the $\mathrm{CO}$ snowline and travels a large distance before they lose all of their $\mathrm{CO}$ ice. However, we do not see any significant enhancement of $\mathrm{CO}$ gas abundance in the inner region of DM Tau and IM Lup.

Although the general radial dependence of $\mathrm{CO}$ depletion is qualitatively similar to the predictions of dust evolution, the absolute magnitudes of depletion found here are much higher than those in dust evolution simulations. We find a factor of 10 or larger $\mathrm{CO}$ depletion in most regions of the four disks, while the simulations of Krijt et al. (2018) predicted a relatively modest depletion (by a factor of 2-6) in most regions of the disk.

\subsubsection{Ways to Increase CO Gas Depletion in Disks}

As discussed above, neither the chemical processing nor the dust growth alone can produce sufficiently large $\mathrm{CO}$ depletion at the observable layer to account for the weak $\mathrm{CO}$ emissions seen in Class II disks.

There are several ways to increase the $\mathrm{CO}$ gas depletion in a disk. The simplest way is a longer dust evolution. The simulations in Krijt et al. (2018) stopped at $1 \mathrm{Myr}$, and the CO depletion gradually increases with time. However, the depletion will eventually reach a steady state when most of the solids become pebbles and stay in the midplane region. The maximum depletion level depends on various disk properties such as the turbulence level and total dust masses.

A more promising way is that there is enough vertical mixing between the atmosphere and the active $\mathrm{CO}$ processing region at the deep layer. The efficiency of this way can be easily seen by comparing the timescales of vertical mixing and chemical processing. The turbulent mixing timescale is given by $t_{\text {mix }} \simeq H^{2} / D \sim\left(\alpha \Omega_{k}\right)^{-1}$, where $\alpha$ is the Shakura-Sunyeav $\alpha$ parameter and $\Omega_{k}$ is the Keplerian angular velocity ( $\mathrm{Xu}$ et al. 2017; Krijt et al. 2018). For a disk around a solar mass star, $t_{\text {mix }} \simeq 10^{5} \mathrm{yr}$ at $100 \mathrm{au}$ for $\alpha=10^{-3}$, and $10^{6} \mathrm{yr}$ for $\alpha=10^{-4}$. The mixing timescales become only $10^{4}$ and $6 \times 10^{4} \mathrm{yr}$ for $\alpha=10^{-3}$ at 20,50 au, respectively. Compared to chemical timescales of CO processing, Bosman et al. (2018) found that the most efficient $\mathrm{CO}$ depletion occurs at regions between $15-30 \mathrm{~K}$ with a typical timescale of $10^{6} \mathrm{yr}$. Therefore, the mixing timescale is in general much shorter or comparable to the chemical timescale of $\mathrm{CO}$ depletion. A coupling of mixing and chemical processes can significantly reduce the $\mathrm{CO}$ gas abundance at the observable layers, especially for regions inside $50 \mathrm{au}$. Therefore, we suggest that a coupled view of physicalchemical evolution is necessary for future investigation of the $\mathrm{CO}$ depletion problem and even general understanding of volatile abundances in planet-forming regions.

\subsection{Effects of Substructures on CO Gas Abundance Profiles}

Recent ALMA long-baseline observations have revealed that many disks show 1-20 au scale substructures in their dust continuum emission (Andrews et al. 2018). Prominent substructures in the gas and dust mass distributions may alter the local CO gas emission. For example, Facchini et al. (2018) found that the gas inside gaps opened by $1-5 M_{J}$ planet becomes colder than the surrounding environment in their thermal-chemical models. The depletion of gas and temperature variation across gaps can affect a radial profile of the $\mathrm{CO}$ abundance derived from a smooth surface density structure such as ones used here.

Although the origins of these dust substructures are still under debate, proposed mechanisms generally predict that substructures in CO line emission would be much narrower and shallower than those of the dust emission (Facchini et al. 2018). Three of the four disks (TW Hya, DM Tau, and IM Lup) only show modest substructures in continuum emission beyond $20 \mathrm{au}$, with up to a factor of 3 depletion inside gaps (Huang et al. 2018b; Kudo et al. 2018). HD 163296 has the most significant substructures among the four disks, showing a factor of 33 depletion inside one of its gaps in the $1.3 \mathrm{~mm}$ continuum (Huang et al. 2018b). But even for the HD 163296 disk, its ${ }^{13} \mathrm{CO}$ and $\mathrm{C}^{18} \mathrm{O}(2-1)$ line observations at 0 .' 2 resolution only show subtle breaks in the slopes rather than clear gaps (Isella et al. 2016). In short, based on the depths and widths of known dust substructures in the four disks and current theoretical predictions, it is unlikely that the order of magnitude variations in $\mathrm{CO}$ abundance profiles are due to substructures alone.

On the other hand, it is important to study how the dust substructures may affect the local chemical substructures and as these local effects may significantly impact the compositions of forming planets. A Large ALMA Cycle 6 program is currently ongoing to study the detailed interplay between dust substructures and local chemical variations.

\subsection{The Distribution of CO Gas Abundance and Disk Properties}

In our small sample, the radial profiles of $\mathrm{CO}$ depletion are diverse. Here we discuss how disk properties might play a role in regulating the radial $\mathrm{CO}$ depletion profile.

HD 163296 is the only Herbig disk in our sample and it shows a very similar depletion pattern to the dust evolution models of Krijt et al. (2018). Assuming a canonical gas-to-dust 
mass ratio of 100 , it has a modest $\mathrm{CO}$ gas depletion in this sample (by a factor of 5 on disk average). Current chemical models suggest that the chemical processing of $\mathrm{CO}$ gas is most efficiently happening at the cold interior of a disk. Herbig disks have a larger fraction of the disk mass above the $\mathrm{CO}$ condensation temperature than that of $\mathrm{T}$ Tauri disks, and therefore they are expected to have less $\mathrm{CO}$ gas processing through the chemical pathways (Bosman et al. 2018). Besides HD 163296, another example of Herbig disk with modest carbon depletion is HD 100546. Kama et al. (2016) analyzed major carbon carriers (CO, C I, C II) for the HD 100546 disk, and concluded that the elemental carbon abundance in its warm molecular layer is close to the interstellar ratio or only modestly depleted, with $\mathrm{C} / \mathrm{H}=(0.1-1.5) \times 10^{-4}$. Considering the old ages of the two Herbig disks (HD $163296 \simeq 12 \mathrm{Myr}$, HD 100546 between 4.1 and $16 \mathrm{Myr}$, Andrews et al. 2018; van der Marel et al. 2019), the chemical and physical processes should already have sufficient time to occur. The fact that the $\mathrm{CO}$ depletion is modest in these two warm disks suggests that temperature may play an important role in $\mathrm{CO}$ depletion processes. A larger sample of Herbig disks can easily confirm or rule out the effect of warm temperature in the $\mathrm{CO}$ depletion observed in protoplanetary disks.

Among the three T Tauri disks studied here, IM Lup is the most massive disk. Although it appears to be relatively young ( $\sim 1 \mathrm{Myr}$ ), its disk-averaged $\mathrm{CO}$ depletion is high, by a factor of 20 (Cleeves et al. 2016). Our model predicts that even with high cosmic-ray ionization rate, the expected $\mathrm{CO}$ depletion is a factor of 2-4. On the other hand, the high $\mathrm{CO}$ depletion in IM Lup is consistent with the expectation that dust evolution is faster in a massive disk.

DM Tau is the coldest disk in our sample but its diskaveraged $\mathrm{CO}$ depletion is just modest. This appears to be inconsistent with chemical processing of $\mathrm{CO}$ happening most efficiently at the cold region of a disk. On the other hand, the DM Tau disk is among the largest disks in both gas lines and (sub)millimeter continuum observations, and its column density is generally lower than those of the other three disks (see Figure 6). The low-density environment can lead to slower dust growth and less $\mathrm{CO}$ depletion.

The TW Hya disk shows the largest CO gas depletion on disk average. In particular, its outer disk region $(>100 \mathrm{au})$ appears to be extremely depleted of $\mathrm{CO}$ (by a factor of 100-300). This extreme $\mathrm{CO}$ depletion in the outermost region is puzzling, as it can be explained by neither chemical processing nor dust evolution. In particular, molecular ion observations toward the TW Hya disks indicate its ionization level due to $\mathrm{CR}$ is quite low $\left(\zeta_{\mathrm{CR}}<10^{-19} \mathrm{~s}^{-1}\right.$ ) (Cleeves et al. 2015). On the dust evolution side, the highest depletion occurs in the outermost region, which is inconsistent with the predictions of dust evolution simulations.

Given these arguments, we suggest that the weak $\mathrm{CO}$ emission beyond $100 \mathrm{au}$ of the TW Hya disk is more likely to be a result of gas dissipation beyond $100 \mathrm{au}$. TW Hya is one of the oldest T Tauri disks (5-10 Myr old) and thus is likely more evolved than other systems. Although its HD (1-0) line flux suggests its total gas disk mass is still high, the HD line mostly traces the relatively warm region inside 50 au (Bergin et al. 2013; Trapman et al. 2017). A lower limit of the gas mass beyond $100 \mathrm{au}$ in the TW Hya disk has been constrained by ratios of resolved CS lines (Teague et al. 2018b). Comparing the difference between the minimum mass and the one used in our model, the CO abundance can be as high as only modestly depleted by a factor of 2-3. In summary, a gas-poor disk beyond $100 \mathrm{au}$ is consistent with current observations of the TW Hya disk.

\section{Summary}

Using well-resolved $\mathrm{CO}$ isotopologue line images and detailed thermo-chemical models, we investigate whether the $\mathrm{CO}$ gas depletion varies with radius in four well-studied protoplanetary disks, and compare these $\mathrm{CO}$ depletion profiles with predictions of chemical processes and dust evolution in protoplanetary disks. In summary, we find:

1. The $\mathrm{CO}$ gas abundance in the warm molecular layer likely varies significantly with radius in these four disks. We find at least one order of magnitude variation within individual disks.

2. Our models show that chemical processing of $\mathrm{CO}$ without vertical mixing even with an ISM level cosmic-ray ionization rate, cannot explain the weak $\mathrm{CO}$ isotopologue lines observed in these disks. The reason is that the ionization driven $\mathrm{CO}$ depletions are active within the deep denser regions close to the midplane of these disks. Without sufficient vertical mixing, the chemical processing cannot cause orders of magnitude weaker $\mathrm{CO}$ isotopologue line emissions.

3. The general trend of the radial profiles of $\mathrm{CO}$ depletion in three disks (except for TW Hya) is qualitatively consistent with predictions of dust evolution models: the outermost disk region generally has higher $\mathrm{CO}$ gas abundance than the intermediate region beyond the midplane $\mathrm{CO}$ snowline. But the observed $\mathrm{CO}$ depletions are a factor of few higher than those in numerical simulations of dust evolution.

4. In the HD 163296 disk, we find its CO gas abundance is slightly enhanced inside its $\mathrm{CO}$ snowline at $70 \mathrm{au}$. Unlike the other three disks, which generally have less depleted $\mathrm{CO}$ in their outermost region, the TW Hya disk shows a distinctive case. Its 100-200 au region is either extremely $\mathrm{CO}$ depleted or already lost a significant amount of gas.

5. We find that neither the dust growth nor chemical processing alone can reproduce the $\mathrm{CO}$ depletion levels seen in these four disks. We suggest that a coupled view of physical and chemical evolution is necessary to investigate the $\mathrm{CO}$ depletion problem and in general to understand what raw materials are available for planet formation at different distances from the central star.

This paper makes use of the following ALMA data: JAO.ALMA\#2013.1.00226.S, 2013.1.00601.S, 2013.1.00798.S, 2015.1.00308.S, and 2016.1.01495.S. ALMA is a partnership of European Southern Observatory (ESO; representing its member states), National Science Foundation (USA), and National Institutes of Natural Sciences (Japan), together with National Research Council (Canada), National Science Council and Academia Sinica Institute of Astronomy and Astrophysics (Taiwan), and Korea Astronomy and Space Science Institute (Korea), in cooperation with Chile. The Joint ALMA Observatory is operated by ESO, AUI/NRAO, and NAOJ. The National Radio Astronomy Observatory is a facility of the National Science Foundation operated under cooperative agreement by Associated Universities, Inc. K.Z., S.K., and K.S. acknowledge the support of 
NASA through Hubble Fellowship grant HST-HF2-51401.001, HST-HF2-51394.001, and HST-HF2-51419.001 awarded by the Space Telescope Science Institute, which is operated by the Association of Universities for Research in Astronomy, Inc., for NASA, under contract NAS5-26555.

\section{Facility: ALMA.}

Software: CASA (McMullin et al. 2007), RADMC3D (Dullemond et al. 2012), RAC2D (Du \& Bergin 2014).

\section{ORCID iDs}

Ke Zhang @ ittps://orcid.org/0000-0002-0661-7517

Edwin A. Bergin (1) https://orcid.org/0000-0003-4179-6394

Kamber Schwarz 나 https://orcid.org/0000-0002-6429-9457

Sebastiaan Krijt (ib https://orcid.org/0000-0002-3291-6887

Fred Ciesla (1) https://orcid.org/0000-0002-0093-065X

\section{References}

Aikawa, Y., \& Herbst, E. 1999, A\&A, 351, 233

Aikawa, Y., Umebayashi, T., Nakano, T., \& Miyama, S. M. 1999, ApJ, 519, 705 Aikawa, Y., van Zadelhoff, G. J., van Dishoeck, E. F., \& Herbst, E. 2002, A\&A, 386, 622

ALMA Partnership, Brogan, C. L., Pérez, L. M., et al. 2015, ApJL, 808, L3 Andrews, S. M., Huang, J., Pérez, L. M., et al. 2018, ApJL, 869, L41 Andrews, S. M., Wilner, D. J., Espaillat, C., et al. 2011, ApJ, 732, 42 Andrews, S. M., Wilner, D. J., Hughes, A. M., et al. 2012, ApJ, 744, 162

Andrews, S. M., Wilner, D. J., Zhu, Z., et al. 2016, ApJL, 820, L40 Ansdell, M., Williams, J. P., Trapman, L., et al. 2018, ApJ, 859, 21 Ansdell, M., Williams, J. P., van der Marel, N., et al. 2016, ApJ, 828, 46 Barenfeld, S. A., Carpenter, J. M., Ricci, L., \& Isella, A. 2016, ApJ, 827, 142 Benz, W., Ida, S., Alibert, Y., Lin, D., \& Mordasini, C. 2014, in Protostars and Planets VI, ed. H. Beuther et al. (Tucson, AZ: Univ. Arizona Press), 691

Bergin, E. A., Aikawa, Y., Blake, G. A., \& van Dishoeck, E. F. 2007, in Protostars and Planets V, ed. B. Reipurth, D. Jewitt, \& K. Keil (Tucson, AZ: Univ. Arizona Press), 751

Bergin, E. A., Cleeves, L. I., Crockett, N., \& Blake, G. A. 2014, FaDi, 168, 61 Bergin, E. A., Cleeves, L. I., Gorti, U., et al. 2013, Natur, 493, 644 Bergin, E. A., Du, F., Cleeves, L. I., et al. 2016, ApJ, 831, 101 Bergin, E. A., \& Williams, J. P. 2018, arXiv:1807.09631

Bergner, J. B., Oberg, K. I., Bergin, E. A., et al. 2019, ApJ, 876, 25

Birnstiel, T., Klahr, H., \& Ercolano, B. 2012, A\&A, 539, A148

Bosman, A. D., Walsh, C., \& van Dishoeck, E. F. 2018, A\&A, 618, A182

Brickhouse, N. S., Cranmer, S. R., Dupree, A. K., Luna, G. J. M., \& Wolk, S. 2010, ApJ, 710, 1835

Calvet, N., D'Alessio, P., Watson, D. M., et al. 2005, ApJL, 630, L185

Cleeves, L. I., Adams, F. C., \& Bergin, E. A. 2013, ApJ, 772, 5

Cleeves, L. I., Bergin, E. A., Qi, C., Adams, F. C., \& Öberg, K. I. 2015, ApJ, 799, 204

Cleeves, L. I., Öberg, K. I., Wilner, D. J., et al. 2016, ApJ, 832, 110

de Gregorio-Monsalvo, I., Ménard, F., Dent, W., et al. 2013, A\&A, 557, A133

Dodson-Robinson, S. E., Evans, N. J. I., Ramos, A., Yu, M., \& Willacy, K. 2018, ApJL, 868, L37

Du, F., \& Bergin, E. A. 2014, ApJ, 792, 2

Dullemond, C. P., \& Dominik, C. 2004, A\&A, 417, 159

Dullemond, C. P., Juhasz, A., Pohl, A., et al. 2012, RADMC-3D: A multipurpose radiative transfer tool, Astrophysics Source Code Library, ascl:1202.015

Eistrup, C., Walsh, C., \& van Dishoeck, E. F. 2016, A\&A, 595, A83

Facchini, S., Pinilla, P., van Dishoeck, E. F., \& de Juan Ovelar, M. 2018, A\&A, 612, A104

Favre, C., Cleeves, L. I., Bergin, E. A., Qi, C., \& Blake, G. A. 2013, ApJL, 776, L38

Flaherty, K. M., Hughes, A. M., Rosenfeld, K. A., et al. 2015, ApJ, 813, 99 Gaia Collaboration, Brown, A. G. A., \& Vallenari, A. 2016, A\&A, 595, A2 Gaia Collaboration, Brown, A. G. A., Vallenari, A., et al. 2018, A\&A, 616, A1
Günther, H. M., Matt, S. P., Schmitt, J. H. M. M., et al. 2010, A\&A, 519, A97 Hayashi, C. 1981, PThPS, 70, 35

Henning, T., Semenov, D., Guilloteau, S., et al. 2010, ApJ, 714, 1511 Huang, J., Andrews, S. M., Cleeves, L. I., et al. 2018a, ApJ, 852, 122

Huang, J., Andrews, S. M., Dullemond, C. P., et al. 2018b, ApJL, 869, L42 Isella, A., Guidi, G., Testi, L., et al. 2016, PhRvL, 117, 251101

Kama, M., Bruderer, S., van Dishoeck, E. F., et al. 2016, A\&A, 592, A83

Kenyon, S. J., \& Hartmann, L. 1995, ApJS, 101, 117

Krijt, S., Ciesla, F. J., \& Bergin, E. A. 2016, ApJ, 833, 285

Krijt, S., Schwarz, K. R., Bergin, E. A., \& Ciesla, F. J. 2018, ApJ, 864, 78

Kudo, T., Hashimoto, J., Muto, T., et al. 2018, ApJL, 868, L5

Long, F., Herczeg, G. J., Pascucci, I., et al. 2017, ApJ, 844, 99

Lynden-Bell, D., \& Pringle, J. E. 1974, MNRAS, 168, 603

Manara, C. F., Rosotti, G., Testi, L., et al. 2016, A\&A, 591, L3

McClure, M. K., Bergin, E. A., Cleeves, L. I., et al. 2016, ApJ, 831, 167

McMullin, J. P., Waters, B., Schiebel, D., Young, W., \& Golap, K. 2007, in ASP Conf. Ser. 376, Astronomical Data Analysis Software and Systems XVI, ed. R. A. Shaw, F. Hill, \& D. J. Bell (San Francisco, CA: ASP), 127

Miotello, A., Bruderer, S., \& van Dishoeck, E. F. 2014, A\&A, 572, A96

Miotello, A., Facchini, S., van Dishoeck, E. F., \& Bruderer, S. 2018, A\&A, 619, A113

Miotello, A., van Dishoeck, E. F., Kama, M., \& Bruderer, S. 2016, A\&A, 594, A85

Miotello, A., van Dishoeck, E. F., Williams, J. P., et al. 2017, A\&A, 599, A113

Molyarova, T., Akimkin, V., Semenov, D., et al. 2017, ApJ, 849, 130

Natta, A., Testi, L., Neri, R., Shepherd, D. S., \& Wilner, D. J. 2001, A\&A, 416, 179

Öberg, K. I., \& Bergin, E. A. 2016, ApJL, 831, L19

Öberg, K. I., Murray-Clay, R., \& Bergin, E. A. 2011, ApJL, 743, L16

Öberg, K. I., van Broekhuizen, F., Fraser, H. J., et al. 2005, ApJL, 621, L33

Pinte, C., Ménard, F., Duchêne, G., et al. 2018a, A\&A, 609, A47

Pinte, C., Padgett, D. L., Ménard, F., et al. 2008, A\&A, 489, 633

Pinte, C., Price, D. J., Ménard, F., et al. 2018b, ApJL, 860, L13

Pollack, J. B., Hubickyj, O., Bodenheimer, P., et al. 1996, Icar, 124, 62

Qi, C., Öberg, K. I., Andrews, S. M., et al. 2015, ApJ, 813, 128

Qi, C., Öberg, K. I., Wilner, D. J., et al. 2013, Sci, 341, 630

Reboussin, L., Wakelam, V., Guilloteau, S., Hersant, F., \& Dutrey, A. 2015, A\&A, 579, A82

Rosenfeld, K. A., Andrews, S. M., Hughes, A. M., Wilner, D. J., \& Qi, C. 2013a, ApJ, 774, 16

Rosenfeld, K. A., Andrews, S. M., Wilner, D. J., Kastner, J. H., \& McClure, M. K. 2013b, ApJ, 775, 136

Salyk, C., Blake, G. A., Boogert, A. C. A., \& Brown, J. M. 2009, ApJ, 699,330

Schwarz, K. R., Bergin, E. A., Cleeves, L. I., et al. 2016, ApJ, 823, 91

Schwarz, K. R., Bergin, E. A., Cleeves, L. I., et al. 2018, ApJ, 856, 85

Schwarz, K. R., Bergin, E. A., Cleeves, L. I., et al. 2019, ApJ, 877, 131

Stammler, S. M., Birnstiel, T., Panić, O., Dullemond, C. P., \& Dominik, C. 2017, A\&A, 600, A140

Swartz, D. A., Drake, J. J., Elsner, R. F., et al. 2005, ApJ, 628, 811

Teague, R., Bae, J., Bergin, E. A., Birnstiel, T., \& Foreman-Mackey, D. 2018a, ApJL, 860, L12

Teague, R., Henning, T., Guilloteau, S., et al. 2018b, ApJ, 864, 133

Trapman, L., Miotello, A., Kama, M., van Dishoeck, E. F., \& Bruderer, S. 2017, A\&A, 605, A69

Umebayashi, T., \& Nakano, T. 1981, PASJ, 33, 617

van Boekel, R., Henning, T., Menu, J., et al. 2017, ApJ, 837, 132

van der Marel, N., Dong, R., di Francesco, J., Williams, J., \& Tobin, J. 2019, ApJ, 872, 112

Williams, J. P., \& Best, W. M. J. 2014, ApJ, 788, 59

Williams, J. P., \& Cieza, L. A. 2011, ARA\&A, 49, 67

Williams, J. P., \& McPartland, C. 2016, ApJ, 830, 32

Wilson, T. L. 1999, RPPh, 62, 143

Xu, R., Bai, X.-N., \& Öberg, K. 2017, ApJ, 835, 162

Yu, M., Willacy, K., Dodson-Robinson, S. E., Turner, N. J., \& Evans, N. J., II 2016, ApJ, 822, 53

Zhang, K., Bergin, E. A., Blake, G. A., et al. 2016, ApJL, 818, L16

Zhang, K., Bergin, E. A., Blake, G. A., Cleeves, L. I., \& Schwarz, K. R. 2017, NatAs, 1, 0130

Zhang, K., Isella, A., Carpenter, J. M., \& Blake, G. A. 2014, ApJ, 791, 42 